\title{
The Importance of Conventional Radiography and Ultrasound in Determining Bone Age
}

\author{
Khizer Razak and GL Meena* \\ Department of Radiodiagnosis, Sardar Patel Medical College, India \\ *Corresponding author: GL Meena, Department of Radiodiagnosis, Sardar Patel Medical College, India
}



\begin{abstract}
Objectives: The purpose of this study is to evaluate the efficacy of ultrasonography data with wrist radiographs taken to determine the extent of pubertal growth and bone age in patients and investigate the usability of ionizing radiation-free ultrasonography instead of conventional radiography.

Methods: In this study, a total of 120 children from 10 to 17 years old (mean age was 168 months \pm 27.5 months) were treated with routine radiographs before orthodontic treatment, and ultrasonographic imaging was performed on the wrists the same day. Researchers examined the phalanges, sesamoid bone, and radial bone distal epiphysis-diaphysis comparatively in each patient by both imaging methods and statistical evaluation.

Results: There was no statistically significant difference between conventional radiography and ultrasonography values at 13 points except for proximal phalanges of the first finger, proximal phalanges of the second finger and radial epiphysis ( $p>0.05)$. The CBA (bone age obtained from conventional radiographs) of the females was found to be larger than their CA (chronological age) and their UBA (ultrasonographic bone age). For males; the means of the CBA, UBA and CA values close to each other. In females and males; there was a strong correlation between the CA, the UBA and the CBA $(\mathrm{p}<0.01)$.
\end{abstract}

Conclusion: Ultrasonography gives detailed information about epiphyseal diaphysis relations. It can be used as an alternative to conventional radiography in the detection of bone age and pubertal growth, owing to the absence of ionizing radiation.

Keywords: Radiology; Imaging; Bone; Age; Ultrasound

\section{Introduction}

Growth and development may vary in chronological age in children of the same age, depending on various genetic and social factors [1-4]. For this reason, skeletal maturation, expressed as bone age; has been accepted as an important criterion for assessing growth and development [5]. Bone age is used for diagnosis and treatment in anthropology, cadavers, and dentistry, paediatric, orthopaedic, and forensic cases in the decision-making process, and in the assessment of criminal and legal responsibility. In dentistry, especially in orthodontic treatment-planned patients, the timing of treatment and selection of the treatment method are gaining importance. Researchers use radiographic methods routinely for bone age determination.

For this purpose, hand-wrist radiographs taken from patients are generally preferred. In hand-wrist radiographs, the right hand is more likely to be exposed to trauma than the left hand, resulting in more reliable values using the left hand [6]. However, due to the possible side effects and damages of X-rays, researchers have always been directed to other radiation-free diagnostic methods. Ultrasonography (USG), a diagnostic method we use in our research, does not contain ionized radiation; it has become very popular in our field in recent years, although its application area in dentistry is usually limited to soft tissue [7,8]. Greulich \& Pyle [9], one of the two most frequently used atlases in the evaluation of wrist radiographs. The second edition of this method, which was used as the GP method, was published in 1959 and is still used today.

In the GP method, evaluations are made by comparing the hand-wrist radiograph obtained from the patients with the standard images stated separately in the atlas for males and females until the age of 18 years. In addition to these, the Tanner-Whitehouse, Gilsanz-Ratibin, Fishman, Bjork, and Grave-Brown methods, automated skeletal bone age assessment, and sonographic age estimation with Bon Age System are also used to determine bone age $[10,11]$. But only radial epiphysis was assessed in this study. This study differs from previous studies on the usability of USG in determining bone age and pubertal growth excretion in adolescent children. The purpose of this study is

(i) To assess the fit between the direct radiography and USG findings from the hand-wrist and

(ii) To investigate whether bone age and pubertal growth excretion are detectable with USG without ionizing radiation. 


\section{Methods and Materials}

\section{Patients}

The study was conducted between May 2016 and June 2017 at Department of Radio diagnosis, Sardar Patel Medical College and attached Prince Bijey Singh Memorial hospital. Patients and parents who participated in the study were informed and approved. In addition, the suitability of the research to scientific ethics has been confirmed. Patients were selected and distributed to the groups so, as to represent their age groups as much as possible. Patients included in the study were divided into eight age groups: 10 years (120-131 months), 11 years (132-143 months), 12 years (144-155 months), 13 years (156-167 months), 14 years (168-179 months), 15 years (180-191 months), 16 years (192-203 months), and 17 years (204-215 months). 120 patients with a range of 120-215 months who applied to our X-ray clinic for routine hand-wrist radiography were included. The inclusion of the patients in the study did not considers gender difference, and each group was formed from as many cases as possible to represent the age group. Patients outside the specified age range, individuals with systemic disease that might affect bone metabolism, people who use drugs that can affect bone metabolism, and patients with a bony pathology or trauma story locally in the left hand-wrist region were excluded.

\section{Study design}

The hand-wrist bones of the patients included in the study were examined with USG and conventional radiography. The evaluations were made with the scoring system specified in the study, and the estimated bone age was determined by using the GP atlas in both methods. A scoring system was established for bone age assessments, selected regions on both radiographs and USG images were scored accordingly, and a comparative assessment of findings was made.

\section{Imaging procedure}

Patients included in the study were examined by the same procedure with the Toshiba Aloka prosound (TM) 300 Platinum Series USG device (Toshiba Corporation, Tokyo, Japan) and 8MHz linear array transducer probe by the same investigator. In the USG examination of the patients, longitudinal examinations were performed with phalanges and sesamoid bones parallel to the probe fingers while the palm was facing up, with the hand on a flat surface to ensure standardization (Figure 1a). The radial epiphysis was evaluated on the longitudinal section with the patient's wrist and fingers twisted while the palm was facing down, keeping the probe parallel to the forearm (Figure 1b). All the hand-wrist radiographs of the individuals involved in the study were performed on the same device (Gendex Dental Systems, Des Plaines, IL) by the same X-ray technician who had at least 5 years of working experience. During the hand-wrist radiographs, the patient was given the appropriate position according to the criteria that the manufacturer of the device had identified. When the patients were positioned, the left hand of the patient was placed on the wrist apparatus to see all fingers and wrist bones. The parameters were set to average $66 \mathrm{kVp}, 5 \mathrm{~mA}, 6 \mathrm{~s}$. The system is directly digital-based, and the images are displayed on the computer screen (Figure 1c).
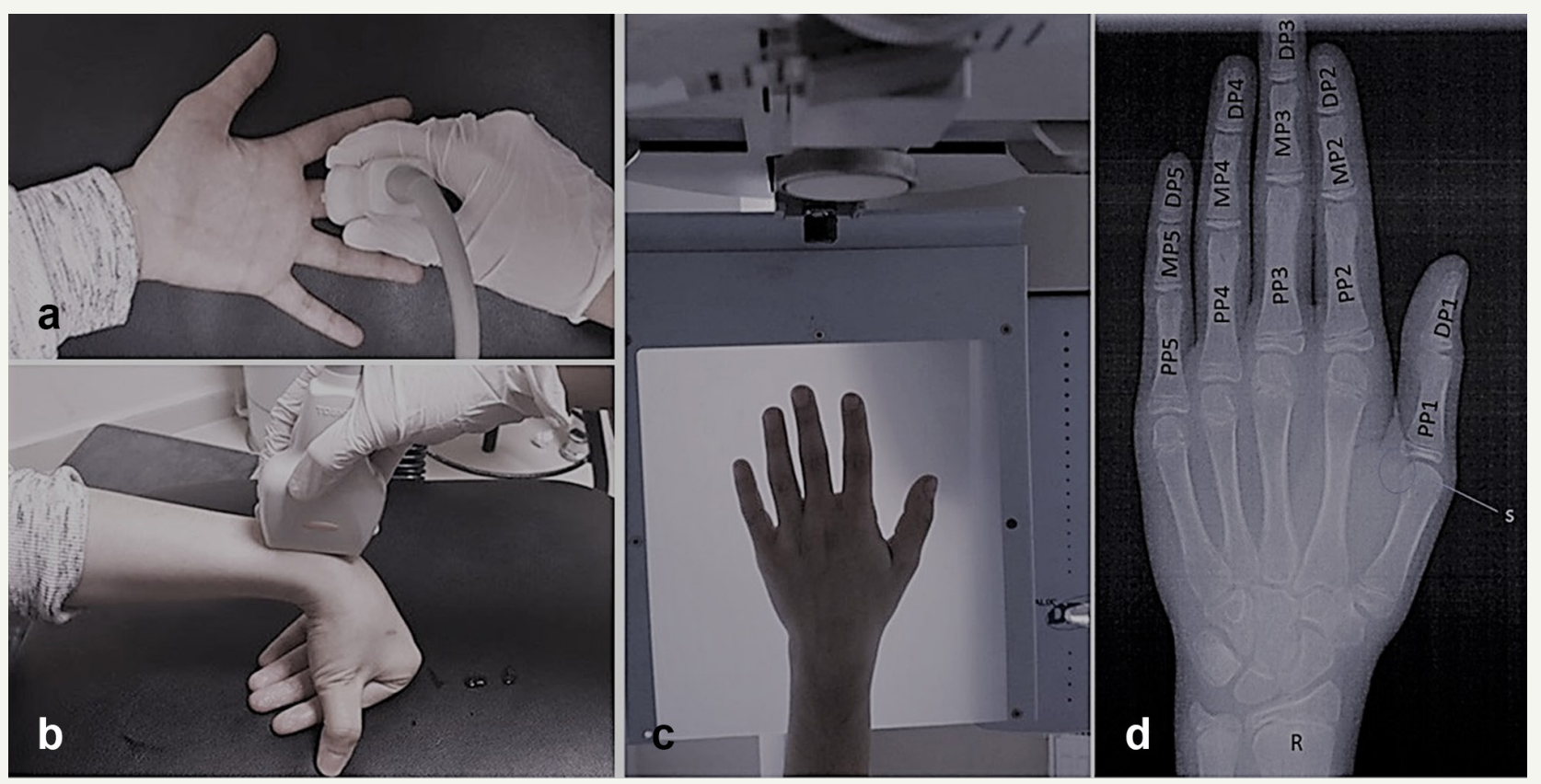

Figure 1:

1a: Healed ulcers of tongue and lip with loss of all the teeth.

$1 \mathrm{~b}$ : Loss of superior part of helix of the external ear (arrow).

1c: Scars on the dorsum of hand (stars) with auto amputation of index and middle finger tips (arrows). 1d: Scar on plantar aspect of heel (arrow).

1e: Rocker bottom deformity of the foot. 


\section{Images evaluation}

As the images were evaluated, USG images were first seen, then hand-wrist radiographs were evaluated. When all the patient's data were collected; to avoid observer bias, all USG images, and then all hand-wrist radiographs (regardless of the patients) were re-evaluated from beginning to end by the same observer. The chronological age was estimated by calculating the time difference between the date of birth of each patient and the hand-wrist radiographs date. In the study, the following points were evaluated (Figure $1 \mathrm{~d}$ ):

1. First finger: Proximal phalanges (PP1), distal phalanges (DP1)

2. Second finger: Proximal phalanges (PP2), middle phalanges (MP2), distal phalanges (DP2)

3. Third finger: Proximal phalanges (PP3), middle phalanges (MP3), distal phalanges (DP3)

4. Fourth finger: Proximal phalanges (PP4), middle phalanges (MP4), distal phalanges (DP4)

5. Fifth finger: Proximal phalanges (PP5), middle phalanges (MP5), distal phalanges (DP5)

6. Radial epiphysis (R)

\section{Sesamoid bone $(\mathrm{S})$}

Another important point in determining pubertal growth is the appearance of the pisiform bone from the carpal bones and the appearance of the hook of the hamate. However, these points were not included in the study due to the difficulty of ultrasonographic imaging. The following scoring system was used to evaluate the images:

1. Epiphysis is smaller than diaphysis and/or no sesamoid bone is seen.

2. Epiphysis equals the diaphysis.

3. Sesamoid is seen.

4. The epiphysis begins to cover the diaphysis (capping).

5. Epiphysis begins to fuse the diaphysis and/or is fused (fusion).

During the evaluation of the images, selected points for study were examined and scored for each epiphysis-diaphysis according to the scoring system described above. While all phalanges and distal radial epiphyses were evaluated separately, imaging and evaluation of the sesamoid bone in USG was performed on the same section as PP1 (Figure $2 \& 3$ ). Two case examples are given in Figure $4 \& 5$.
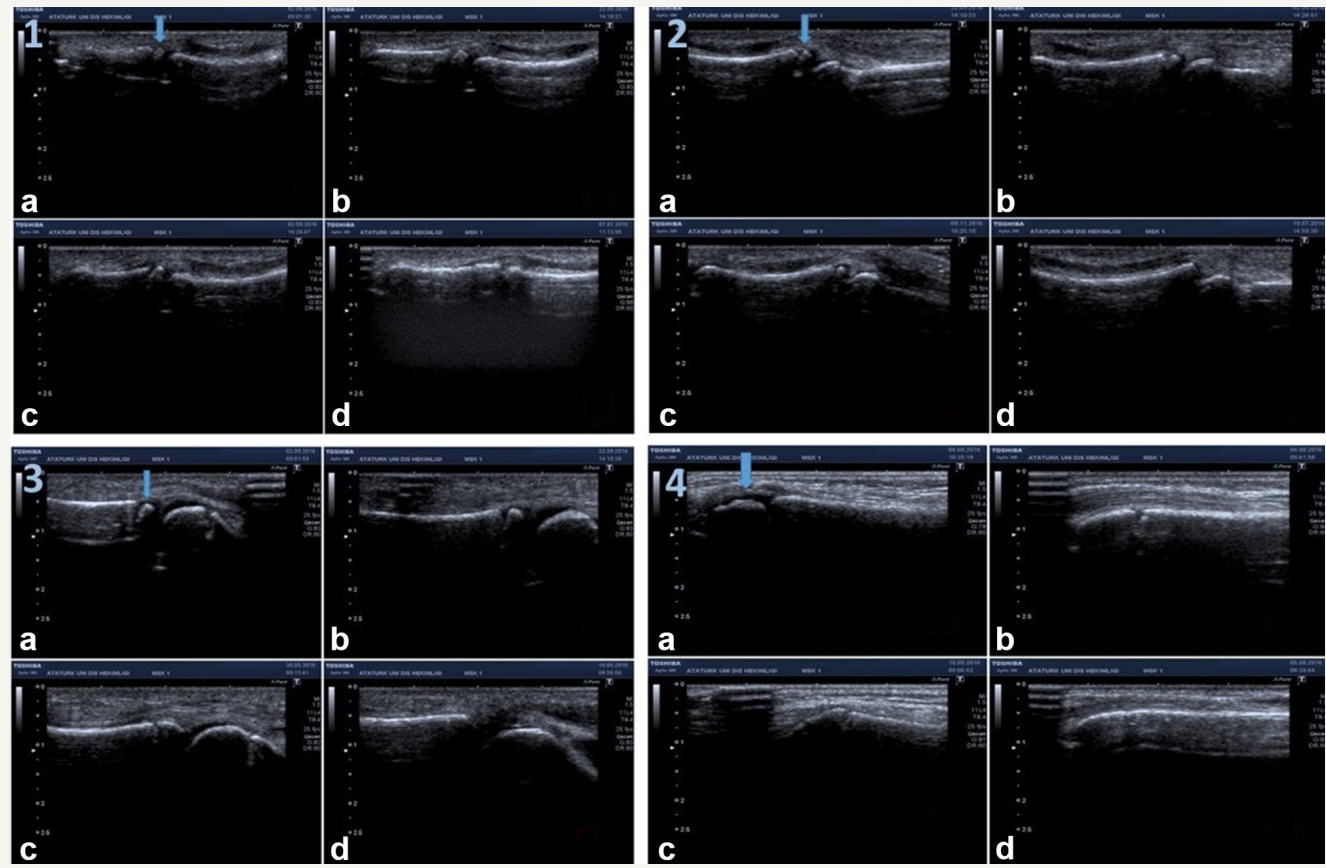

Figure 2: Arrows sign indicates epiphysis; (1) evaluation of distal phalanges, (2) evaluation of middle phalanges, (3) evaluation of proximal phalanges, (4) evaluation of distal radial epiphysis; (a) the epiphysis is smaller than diaphysis. (b) The epiphysis equals the diaphysis. (c) The epiphysis begins to cover the diaphysis (capping). (d) The epiphysis begins to fuse the diaphysis and/or fused (fusion).

\section{Statistical analysis}

A single investigator evaluated all parameters. To determine the investigator's intra observer reliability, $20 \%$ of the assessed images were randomly selected and evaluated by the same investigator a second time after 2 weeks, intraview reliability was found to be $96 \%$. In addition, a second researcher was consulted in the case where the researcher hesitated to evaluate. The one case in which consensus was not provided was not included in the study $(\mathrm{n}=1)$.

The data used in this study were analyzed using the SPSS Statistics 20 package program (Statistical Package for Social Sciences, 
IBM Corp., Armonk, NY). The Shapiro-Wilk test, a non-parametric test, was used for the normality test to determine whether the data fit the normal distribution. Because the data did not fit the normal distribution; the non-parametric Mann-Whitney U test was used in evaluating phalanges, radial joints, and sesamoid bones. The Pear- son's correlation test was used to evaluate the correlation between chronological age (CA), ultrasonographic bone age (UBA), and bone age obtained from conventional radiographs (CBA) within gender (between females and males).


Figure 3: Imaging of the sesamoid bone in USG. $(\mathrm{a}, \mathrm{b})$ The sesamoid bone is seen; The sesamoid bone has not yet begun to be seen (the thin arrow indicates the epiphysis, the thick arrow indicates the sesamoid). USG, ultrasonography.

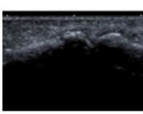

DP1: capping

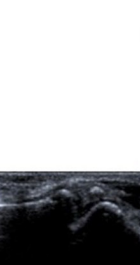

PP1: capping

Sesamoid: seen

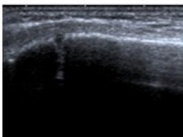

Radius: small

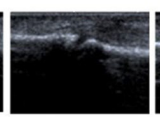

DP2: capping

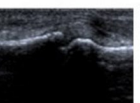

MP2: capping

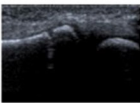

PP2: equal

PP3: capping

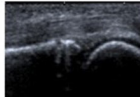

(2)

PP5: capping

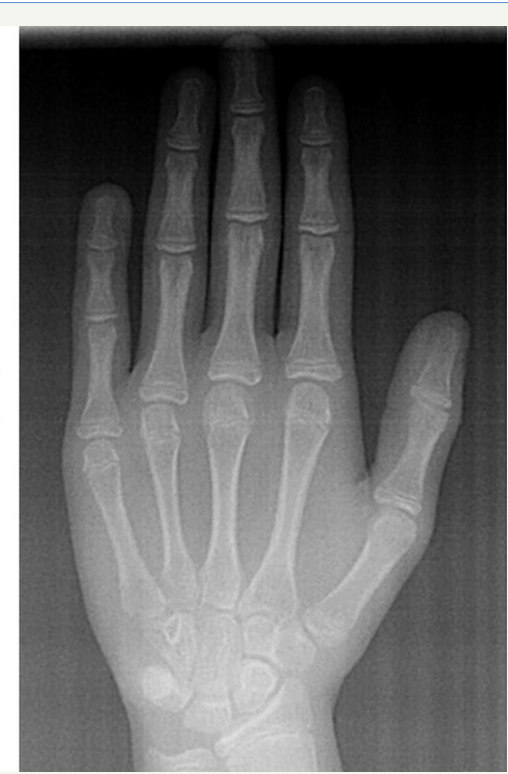

Figure 4: Sample case; female; Chronological age: 12 years 5 months; Conventional bone age: 13 years; Ultrasonographic bone age: 13 years.

\section{Results}

In this study, the hand-wrist bones of 120 patients were evaluated; 38 cases $(31.7 \%)$ were males and $82(68.3 \%)$ were females. The chronological age of the children varied between 10 and 17 years (120-215 months); mean age was $168 \pm 27.5$ months. The average chronological age of the females was $172 \pm 27.2$; the average chronological age of males was $160 \pm 26.5$ months. Statistical distribution of scores obtained from phalanges, sesamoid bones, and distal radial epiphyses on radiographs and USG scans were assessed.
There was a statistically significant difference between the study points in PP1, PP2, and radial epiphysis ( $\mathrm{p}<0.05)$; no significant difference was found between all DPs, all MPs, sesamoid bones, PP3s, PP4s, and PP5s ( $p>0.05$ ). The CBA of the females was found to be larger than their CA and their UBA. For males; the means of the CBA, UBA and CA values close to each other. The relationship between CA, UBA, and CBA was investigated by the Pearson's correlation test. In females; there was a strong correlation between the CA and the CBA ( $r=0.864)$, between the UBA and the CBA $(r=0.847)$, 
and between the CA and UBA $(\mathrm{r}=0.780)(\mathrm{p}<0.01)$. In males; there was a strong correlation between the CA and UBA ( $r=0.891)$, be- tween the CA and the CBA ( $\mathrm{r}=0.817)$, between the UBA and the CBA $(\mathrm{r}=0.745)(\mathrm{p}<0.01)$.

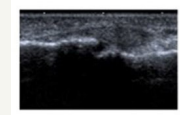

DP1: fusion

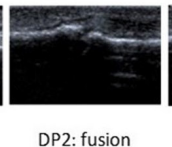

DP2: fusion



MP2: fusion

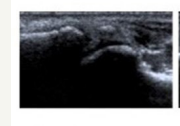

PP1: fusion

Sesamoid: seen

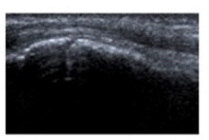

Radius: capping

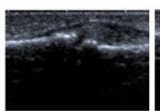

DP3: fusion

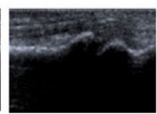

MP3:capping

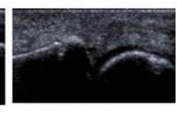

PP3: fusion



DP4: fusion

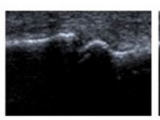

MP4: fusion

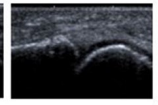

PP4: fusion

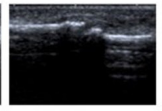

DP5: fusion

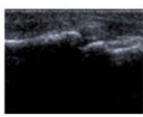

MP5: fusion

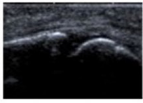

PP5: fusion

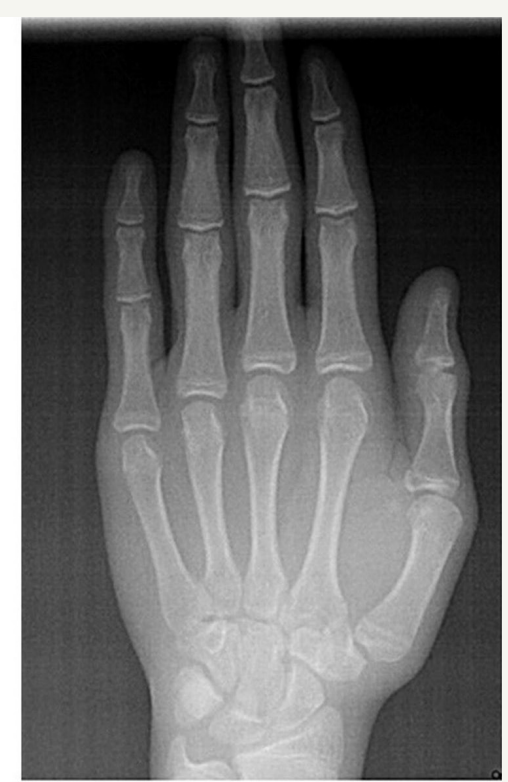

Figure 5: Sample case; male; Male; Chronological age: 16 years 9 months; Conventional bone age: 16 years; Ultrasonographic bone age: 16 years 6 months.

\section{Discussion}

Many areas of bone age determination have important places, and in dentistry it is important to determine pubertal growth. Hand-wrist radiographs, which are among the many methods recommended for evaluating skeletal maturation, are easily applied and routinely used in this regard. Radiographs are simple, have a wide range of uses with reasonable cost and relative reliability, but the cumulative effect of ionizing radiation on children causes many researchers anxiety [12]. In this study, USG, which is a fast, cheap, non-ionizing and easily accessible method, evaluated the relationship between sesamoid bone and epiphysis-diaphysis.

To investigate the efficacy and applicability of USG in bone age determination, the data were compared with hand-wrist radiographs, which were accepted as the gold standard for bone age determination when used with the GP atlas. Studies on the assessment of bone age with radiographs have resulted in a marked change in the maturation of carpal bones and a delayed bone age when carpal bones are considered $[13,14]$. However, Carpenter \& Lester [15] showed that skeletal age determination based on metacarpals and phalanges is closer to chronological age than radius and carpal bones. Bilgili et al. [16] also evaluated carpal bones and epiphyses of phalanges and metacarpal bones in children up to 6 years of age in USG.

However, they pointed out that the image of the wrist bones is a cumulative measure rather than the actual size. USG is an accessible technique that provides real-time, multi-plane images with the ability to compare contralateral [16]. Unlike radiography, the absence of ionizing radiation provides a unique opportunity for detailed and long-term USG examinations [17]. USG can identify centres of ossification with characteristic echogenicity and acoustic shadow [12]. Nessi et al. [18] reported that USG is an easy, valuable, and non-ionizing method for imaging skeletal maturation of the ossification centres of the hand and wrist (sesamoid bone and DP3).

Carpenter \& Lester [15] stated that if the bone age is detected in children under 10 years of age, the entire hand must be considered, and if the carpal bones are weighted, they may reach the point where bone age may be the subject of forward or reverse readings. Radiography or USG has been proposed as an ideal method, with a balanced approach involving all bones of the distal extremity of the upper extremity, including distal radius and ulna, carpal bones, metacarpal bones, and phalanges [16]. In addition, it has been stated that maturation in carpal bones may differ between patients, and that metacarpal bones and phalanges may be more accurate in predicting bone age $[15,16]$. Some researchers have also worked with specially designed USG devices to automatically determine the bone age in children without operator intervention $[11,19,20]$.

The BonAge system, which is a method of determining the age with sound waves, has not been found in clinical use because of its low-accuracy evaluation of only the distal radial joint $[17,20]$. The studies in the literature on bone age determination from USG are usually performed on younger children at the onset of calcification of wrist bones and not yet completed $[12,19,21]$. However, most of the skeletal age assessment in clinical practice in dentistry is performed on children aged 5-18 years [19]. For this reason, the availability of any method that is an alternative to plain radiographs is very important in this age range. This study was based on the usability of USG in the determination of bone age and pubertal growth in the adolescent children, unlike the age group of previous studies. At the same time, not only radial epiphysis but also phalanges are evaluated with USG. This study was the first study with conventional USG in this age group. 
Algazzar et al. [21] conducted a study on 3 month and 6-yearold 5 months children. They formed a table with the GP atlas, calculated the bone age from hand-wrist USG, and compared it with plain radiograph bone age. As a result, they reported that the USG gave the right results in this regard. In a study by Bilgili et al. [16] they constructed a ultrasonographic template under the guidance of the GP atlas and evaluated the images according to this template. They found a high correlation between ultrasonographic and radiographic results in detecting skeletal age in children and reported that there was no statistical difference between the two methods when a deviation of \pm 2 months was accepted as normal.

Ultimately, the ultrasonographic version of the GP atlas has been proposed as an alternative to radiography in detecting bone age without exposure to radiation. But this template created by Bilgili et al. [16] is not suitable for use in all age groups. In a recent study, Daneff et al. [12] evaluated the bone age with conventional USG in healthy infants aged 1-24 months and concluded that conventional USG could identify hand and wrist ossification centers and could be used as a non-invasive follow-up tool for patients with growth problems. Khan et al. [22] reported a low correlation between USG and the GP atlas and radiography in their study of a population of patients with growth disorders and did not consider USG as a valid alternative when determining bone age. On the other hand, Mentzel et al. [19] reported a strong correlation between the GP method and USG in the study of the distal radius and the ulna with the same device.

Both studies, unlike our patient group, included patients with confirmed or suspected growth disorders $[17,19]$. Hajalioghli et al. [23] contrary to the results of Khan et al. [17] shows that USG, compared with radiography, tends to give results close to the actual bone age. They also said that the conflicting results between these two studies were due to Khan et al. [17] using an automatic sonography device. Since this study was conducted on healthy children, no information was available on how effective USG was in detecting growth disorders. However, the study's findings expressed that it is appropriate to use USG as an alternative to radiography in the age determination of healthy patients.

Examination of the bone maturation with USG is practically feasible, because it does not take much time. Each finger, sesamoid, and radial epiphysis may be visualized in a longitudinal plane by an expert radiologist within 2-3min, depending on the person. Handwrist USGs can also be preserved like conventional grafts, and the GP atlas can be used to evaluate hand-wrist USG. Using the GP atlas, USG data can be used to predict the patient's pubertal growth and bone age. In this study, it was shown that using USG along with the GP atlas, which researchers have been using for years, was shown to be able to determine bone age and pubertal growth overrun. In this study, both methods showed a high correlation between estimated bone ages and chronological age.

Determining the extent of pubertal growth in dentistry is the greatest goal of radiographs. It is also valid for the USG to reach the capping phase of the MP3, which is the indicator of pubertal growth breakthrough to reach the peak, and to start to see the sesamoid. It is possible to say that patients who show sesamoid bone and MP3 capping in USG of the hand-wrist, the other epiphyseal diaphysis associations are considered, and reach the peak of pubertal excretion.

Patients included in this study were observed to have a good fit between the data obtained from the radiographs and the data obtained from USG. No statistical differences were observed in all other values except PP1, PP2, and radial epiphysis. PP1, PP2, and radial epiphysis were thought to differ between the two methods due to reasons such as technical precision or application angle. Because USG can detect even small epiphysis- diaphysis openings that are not visible in radiography, the USG may sometimes display an earlier stage than conventional radiography. In this study with a small patient population, the low number of patients can be considered as a limit. However, this study confirmed the idea that even in the current patient population, USG can replace the conventional radiographic method in determining the extent of pubertal growth in children.

It is considered necessary to work with more patient groups to make a more systematic determination of age in USG and to minimize the differences depending on the practitioner by forming an ultrasonographic atlas based on chronological age. Ultrasonographic imaging, although not time-consuming, is required by experienced radiologists, and the results can vary between observers, limiting the use of USG. Another weakness of this study is that both the ultrasound and the radiographs were interpreted by the same observer, thus inducing a risk for observer bias.

Although not present in this study, it was thought that the abnormal calcification of epiphyses or morphological anomalies could be difficult to diagnose by USG. In addition, it may be difficult to identify skeletal anomalies that may help in the diagnosis of growth disorders with USG.

\section{Conclusion}

It can be concluded that hand-wrist radiographs, which are traditional bone age determination methods, can be replaced with USG in the determination of bone age and especially in determining the extent of pubertal growth in the field of dentistry. When both techniques are available, it is advisable to decide which method is appropriate by making a profit-loss account.

\section{References}

1. Gilli G (1996) The assessment of skeletal maturation. Horm Res 45(Suppl 2): 49-52.

2. Mora S, Boechat MI, Pietka E, Huang HK, Gilsanz V (2001) Skeletal age determinations in children of European and African descent: applicability of the greulich and pyle standards. Pediatr Res 50(5): 624-628.

3. Groell R, Lindbichler F, Riepl T, Gherra L, Roposch A, et al. (1999) The reliability of bone age determination in central European children using the Greulich and Pyle method. Br J Radiol 72(857): 461-464. 
4. Dahlberg PS, Mosdol A, Ding KY, Bleka O, Rolseth V, et al. (2017) Agreement between chronological age and bone age based on the Greulich and Pyle atlas for age estimation: a systematic review. Knowledge Centre for the Health Services at The Norwegian Institute of Public Health, Oslo, Norway.

5. Tanner JM, Whitehouse RH (1975) Assessment of skeletal maturity and prediction of adult height (TW2 Method). Academic Press, New York, USA.

6. Roche AF (1967) A study of skeletal maturation in a group of Melbourn children. Aust Paediatr J 3(3): 123-127.

7. Block B (2004) The practice of ultrasound, a step by step guide to abdominal scanning. Thieme Medical Publishers, New York, USA.

8. Nielsen TJ, Lambert MJ (2003) Physics and instrumentation. In: Mateer JR (Ed.), Emergency ultrasound. McGraw-Hill, New York, USA, pp. 45-66.

9. Greulich WW, Pyle SI (1959) Radiographic atlas of skeletal development of the hand-wrist. ( $2^{\text {nd }}$ edn). Stanford University Press, Carlifonia, USA.

10. Satoh M (2015) Bone age: assessment methods and clinical applications. Clin Pediatr Endocrinol 24(4): 143-152.

11. Shimura N, Koyama S, Arisaka O, Imataka M, Sato K, et al. (2005) Assessment of measurement of children's bone age ultrasonically with sunlight bonage. Clin Pediatr Endocrinol 14: S24_17-S24_20.

12. Daneff M, Casalis C, Bruno CH, Bruno DA (2015) Bone age assessment with conventional ultrasonography in healthy infants from 1 to 24 months of age. Pediatr Radiol 45(7): 1007-1015.

13. Garn SM, Rohmann CG (1960) Variability in the order of ossification of the bony centers of the hand and wrist. Am J Phys Anthropol 18(3): 219 230.

14. Garn SM, Rohmann CG (1962) Parent-child similarities in hand-wrist ossification. Am J Dis Child 103: 603-607.
15. Carpenter CT, Lester EL (1993) Skeletal age determination in young children: analysis of three regions of the hand/wrist film. J Pediatr Orthop 13(1): 76-79.

16. Bilgili Y, Hizel S, Kara SA, Sanli C, Erdal HH, et al. (2003) Accuracy of skeletal age assessment in children from birth to 6 years of age with the ultrasonographic version of the Greulich-Pyle atlas. J Ultrasound Med 22(7): 683-690.

17. Khan KM, Miller BS, Hoggard E, Somani A, Sarafoglou K (2009) Application of ultrasound for bone age estimation in clinical practice. J Pediatr 154(2): 243-247

18. Nessi R, Garattini G, Bazzini E, Zaffaroni R, Lazzerini F (1997) Ultrasonography assessment of ossification foci of the wrist and pubertal growth spurt. Radiol Med 94(1-2): 43-46.

19. Mentzel HJ, Vilser C, Eulenstein M, Schwartz T, Vogt S, et al. (2005) Assessment of skeletal age at the wrist in children with a new ultrasound device. Pediatr Radiol 35(4): 429-333.

20. Scanderbeg AC, Sacco MC, Gialloreti LE, Fraracci L (1998) Skeletal age assessment in children and young adults: comparison between a newly developed sonographic method and conventional methods. Skeletal Radiol 27(5): 271-277.

21. Algazzar HY, Nancy M, Zeidan NM (2005) Validity of the ultrasonographic version chart of the Greulich-Pyle method in assessment of skeletal age. Egypt J Radiol Nucl Med 36: 633-643.

22. Khan K, Elayappen AS (2012) Bone growth estimation using radiology (Greulich-Pyle and tanner-whitehouse methods). In: Preedy VR (Ed.), Handbook of growth and growth monitoring in health and disease. Springer, New York, USA, pp. 2937-2953.

23. Hajalioghli P, Tarzamni MK, Arami S, Fouladi DF, Ghojazadeh M (2015) The utility of ultrasonographic bone age determination in detecting growth disturbances; a comparative study with the conventional radiographic technique. Skeletal Radiol 44(9): 1351-1356.
Creative Commons Attribution 4.0

International License

For possible submissions Click Here

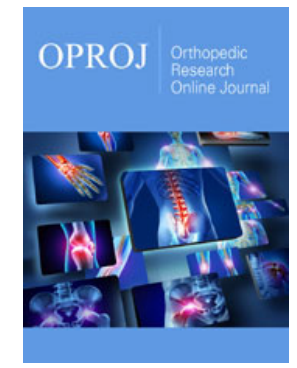

Orthopedic Research Online Journal

\section{Benefits of Publishing with us}

- High-level peer review and editorial services

- Freely accessible online immediately upon publication

- Authors retain the copyright to their work

- Licensing it under a Creative Commons license

- Visibility through different online platforms 